\title{
Identification of hub genes and key pathways of dietary advanced glycation end products-induced non-alcoholic fatty liver disease by bioinformatics analysis and animal experiments
}

\author{
JIAO WANG ${ }^{1}$, HONGHONG LIU ${ }^{1}$, GUIJIAO XIE ${ }^{1}$, WEI CAI $^{2}$ and JIXIONG XU ${ }^{1}$ \\ ${ }^{1}$ Department of Endocrinology and Metabolism, First Affiliated Hospital of Nanchang University; \\ ${ }^{2}$ Department of Medical Genetics and Cell Biology, Medical College of Nanchang University, \\ Nanchang, Jiangxi 330006, P.R. China
}

Received July 22, 2019; Accepted November 18, 2019

DOI: $10.3892 / \mathrm{mmr} .2019 .10872$

\begin{abstract}
Non-alcoholic fatty liver disease (NAFLD) is a common chronic liver disease. Advanced glycation end products (AGEs) negatively affect the liver and accelerate NAFLD progression; however, the underlying mechanisms remain unclear. The present study aimed to examine the effect and mechanism of dietary AGEs on the mouse liver using bioinformatics and in vivo experimental approaches. Gene expression datasets associated with NAFLD were obtained from the Gene Expression Omnibus and differentially expressed genes (DEGs) were identified using GEO2R. Functional enrichment analyses were performed using the Database for Annotation, Visualization and Integrated Discovery and a protein-protein interaction network for the DEGs was constructed using the Search Tool for the Retrieval of Interacting Genes database. MCODE, a Cytoscape plugin, was subsequently used to identify the most significant module. The key genes involved were verified in a dietary AGE-induced non-alcoholic steatohepatitis (NASH) mouse model using reverse transcription-quantitative PCR (RT-qPCR). The 462 DEGs associated with NAFLD in the two datasets, of which 34 overlapping genes were found in two microarray datasets. Functional analysis demonstrated that the 34 DEGs were enriched in the 'PPAR signaling pathway', 'central carbon metabolism in cancer', and 'cell adhesion molecules (CAMs)'. Moreover, four hub genes (cell death-inducing DFFA-like effector a, cell death-inducing DFFA-like effector c, fatty acid-binding protein 4 and perilipin 4) were identified from a protein-protein interaction network and were verified using RT-qPCR in a mouse model of NASH. The results suggested that AGEs and their receptor axis may be
\end{abstract}

Correspondence to: Dr Jixiong Xu, Department of Endocrinology and Metabolism, First Affiliated Hospital of Nanchang University, 17 Yongwaizheng Street, Nanchang, Jiangxi 330006, P.R. China

E-mail: jixiong.xu@ncu.edu.cn

Key words: differentially expressed genes, non-alcoholic fatty liver disease, advanced glycation end products, mice model involved in NAFLD onset and/or progression. This integrative analysis identified candidate genes and pathways in NAFLD, as well as DEGs and hub genes related to NAFLD progression in silico and in vivo.

\section{Introduction}

Non-alcoholic fatty liver disease (NAFLD) is one of the most common chronic liver diseases worldwide and is an important global public health issue (1). NAFLD is observed in metabolic syndrome, and is typically accompanied by hepatic insulin resistance, dyslipidemia and hyperglycemia (2); it consists of a wide spectrum of liver pathologies ranging from simple steatosis to non-alcoholic steatohepatitis (NASH) with fibrosis, which may progress to liver cirrhosis and even hepatocellular carcinoma (3). Current non-pharmacological therapies for NAFLD are mainly based on physical activity and dietary modifications, such as the consumption of a Mediterranean diet, which has been demonstrated to be effective (4-6).

Advanced glycation end-products (AGEs) are products of non-enzymatic reactions of reducing sugars that have free amino groups of nucleotides, lipids and peptides/proteins (7). AGEs may be formed endogenously as a part of normal metabolism and aging, particularly under conditions of high plasma glucose, or exogenously, entering the body through the ingestion of certain foods, such as barbecued food, cheeses, and foods high in fat and sugar. Dry-heat-cooked foods are typical in the modern diet; these contain high levels of AGEs and their consumption contributes to high levels of AGEs in the body (8). Notably, the consumption of modern diets containing excessive AGEs has increased in the past 20 years (9). The accumulation of AGEs can exert detrimental effects and accelerate the progression of AGE-related damage, such as diabetes, atherosclerosis and NAFLD (10-12); however, the exact mechanisms underlying the effects of dietary AGEs on NAFLD remain largely unknown.

Microarray technology enables the determination of mRNA profiles associated with human disease and provides a comprehensive, unbiased approach to systematically analyze disease processes, including NAFLD (13). Additionally, some studies have revealed that the induction of a high-fat or NASH 
diet may promote alterations at the genome level, which are involved in NAFLD (13-15). Accordingly, integrative analyses of genes and pathways associated with modern diet-induced liver injury may provide an insight into therapeutic targets and diagnostic biomarkers for NAFLD.

The present study aimed to analyze differentially expressed genes (DEGs) in the liver tissues from mice with western diet-induced liver disease and those that were administered a regular diet using data downloaded from the Gene Expression Omnibus (GEO). Hub genes were screened from a protein-protein interaction (PPI) network and were verified using reverse transcription-quantitative PCR (RT-qPCR) in a mouse model of NASH. This integrative analysis identified candidate genes and pathways in NAFLD, as well as DEGs and hub genes related to NAFLD progression in silico and in vivo.

\section{Materials and methods}

Microarray data. The data were screened and analyzed by two contributors using the following criteria for data analysis: i) The mouse strain was C57BL/6; and ii) comparison was conducted between NAFLD groups [high fat diet (HFD) or NASH diet, which mimics a modern diet] and normal diet (ND) groups (negative control). Datasets GSE57425 and GSE52748 were acquired from the GEO (http://www.ncbi. nlm.nih.gov/geo; version 2.0) database for analysis $(16,17)$. In the GSE52748 dataset, C57BL/6 mice (male; age, 14 weeks) were fed a ND or NASH-induced diet enriched with sucrose, cholesterol and saturated fatty acids for 12 weeks. In the GSE57425 dataset, C57BL/6 mice (male; age, 8 weeks) were fed a ND or HFD containing $60 \mathrm{kcal} \%$ of fat for 12 weeks. The probes were converted into the corresponding gene symbols according to annotation information provided by the platform.

Identification of DEGs. DEGs were analyzed using GEO2R (http://www.ncbi.nlm.nih.gov/geo/geo2r; version 2.19.4), an online web tool that allows users to compare two or more datasets in a GEO series (18). Probe sets without corresponding gene symbols or genes with $>1$ probe set were averaged. Samples with an absolute value of log fold-change $>1$ and $\mathrm{P}<0.05$ were considered DEGs.

Functional enrichment analysis. To investigate the biological characteristics and functional enrichment of candidate DEGs, functional enrichment analysis was performed using Database for Annotation, Visualization and Integrated Discovery (https://david.ncifcrf.gov/; version 6.8). Results with $\mathrm{P}<0.05$ were considered significant. Additionally, Circos, a visualization software (version 0.1.1) for comparative genomics (19), was applied to identify overlapping genes from the input gene lists and shared GO terms, and a Venn diagram was plotted using an online tool (http://bioinformatics.psb.ugent.be/webtools/Venn/; version 1.0).

PPI network construction and module analysis. A PPI network for DEGs was constructed using the Search Tool for the Retrieval of Interacting Genes (STRING) database (https://string-db.org/cgi/; version 11.0). Interactions with a combined score of $>0.4$ were considered significant. The results were visualized using Cytoscape software (version 3.7.1) (20). MCODE, a Cytoscape plugin, was used to identify the most significant module. The criteria for selection were as follows: MCODE score $\geq 3$, degree cutoff $=2$, node score cutoff $=0.2$ and $\max$ depth $=100$.

Animal studies. The experimental protocol of this study was approved by the Research Committee of the First Affiliated Hospital of Nanchang University (Nanchang, China). In total, 20 C57BL/6J mice (male; age, 12 weeks; weight, 25-28 g) were purchased from Hunan SJA Laboratory Animal Co., Ltd., and maintained at $12 \mathrm{~h}$ light/dark cycle with free access to food and water in a temperature- and humidity-controlled environment of $20-24^{\circ} \mathrm{C}$ and $45-55 \%$ humidity. Mice were divided into two groups, a ND group and high AGE diet group, and fed normal chow and a baked diet, respectively, for 24 weeks. Dietary AGEs were produced by baking the food at $120^{\circ} \mathrm{C}$ for $15 \mathrm{~min}$. The AGE content of the food was measured using ELISA (21), which demonstrated that baking the chow diet increased AGE levels by $>2$-fold from $3,194 \pm 330$ to $6,639 \pm 750 \mathrm{ng} / \mathrm{g}$. The NASH mouse model was established using a baked diet that contains high levels of AGEs. The mice were fed a baked diet for 24 weeks, and exhibited a NASH phenotype with steatosis, liver injury and increased expression of inflammatory and fibrogenic factors. These mice were sacrificed after the 24 weeks of feeding, and then the livers were harvested for assessment.

Liver injury and histopathology. Blood samples were collected from the femoral artery and were centrifuged at 2,500 x g for $15 \mathrm{~min}$ at $4^{\circ} \mathrm{C}$ to obtain serum. Serum alanine aminotransferase (ALT) and aspartate aminotransferase (AST) levels were evaluated to assess liver injury using a Hitachi 7600 biochemical analyzer (Hitachi, Ltd.). Hematoxylin \& eosin (H\&E) staining was performed in paraffin-embedded liver tissue. The livers were fixed in $4 \%$ paraformaldehyde solution for $24 \mathrm{~h}$ at room temperature and then dehydrated. Sections were then embedded with paraffin, cut into serial sections (thickness, $5 \mu \mathrm{m}$ ), dewaxed and rehydrated with graded ethyl $(100,95,80,70$, and $0 \%)$. For H\&E staining, the slides were first incubated with hematoxylin (cat. no. G1120; Beijing Solarbio Science \& Technology Co., Ltd.) for 6 min at room temperature and then washed with $1 \%$ ethanol hydrochloride for $10 \mathrm{sec}$. After washing with water, the slides were stained with $1 \%$ eosin for $3 \mathrm{~min}$ and dehydrated with graded ethyl concentrations. Vacuoles were considered to have steatosis, as shown by H\&E staining (22). Oil red O staining was performed on frozen tissue, as previously described (23). Liver tissues were cryosectioned (thickness, $5 \mu \mathrm{m}$ ), fixed in $10 \%$ formalin solution at room temperature for $10 \mathrm{~min}$ and dipped in $60 \%$ isopropanol for $3 \mathrm{~min}$ at room temperature. The slides were then immersed in $1 \%$ ORO solution for $3 \mathrm{~min}$ at room temperature and washed in $60 \%$ isopropanol followed by distilled water. The slides were counterstained with Mayer hematoxylin (cat. no. G1080, Beijing Solarbio Science \& Technology Co., Ltd.) for $30 \mathrm{sec}$ at room temperature and were mounted onto glycerin gelatin. The collagen content of the liver was assessed by Sirius red staining, as described previously (24). Sections were cut at $5 \mu \mathrm{m}$ and dewaxed in xylene, rehydrated in decreasing concentrations of ethanol, 
A

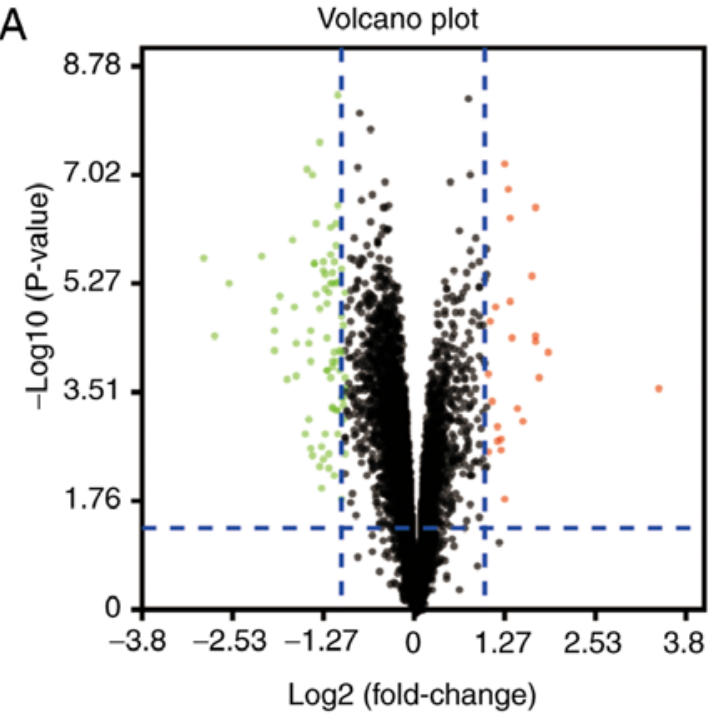

C

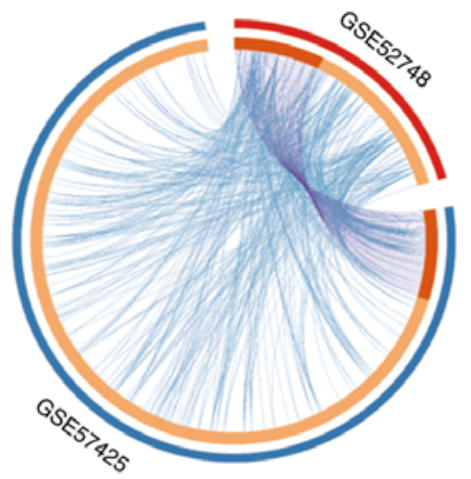

E

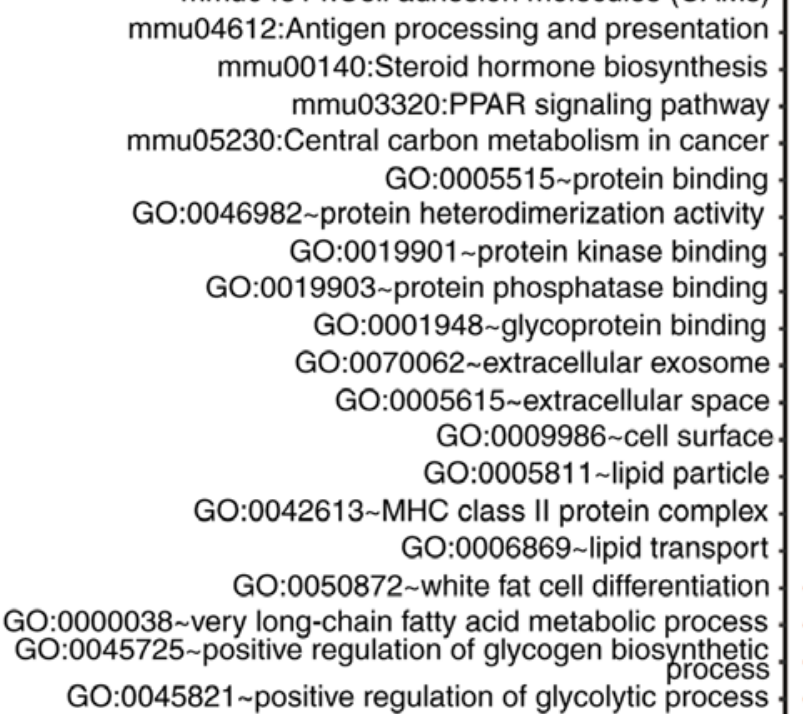

B

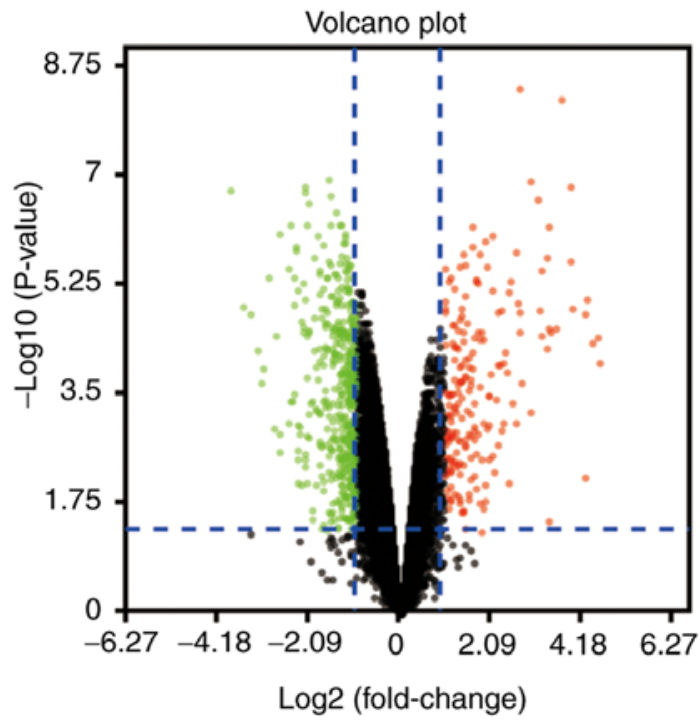

D

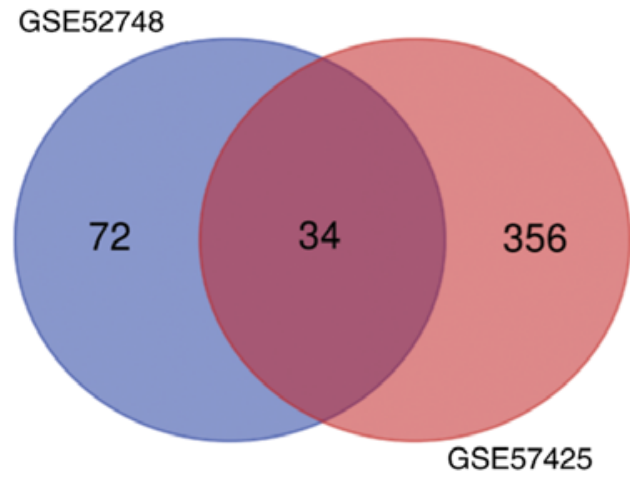

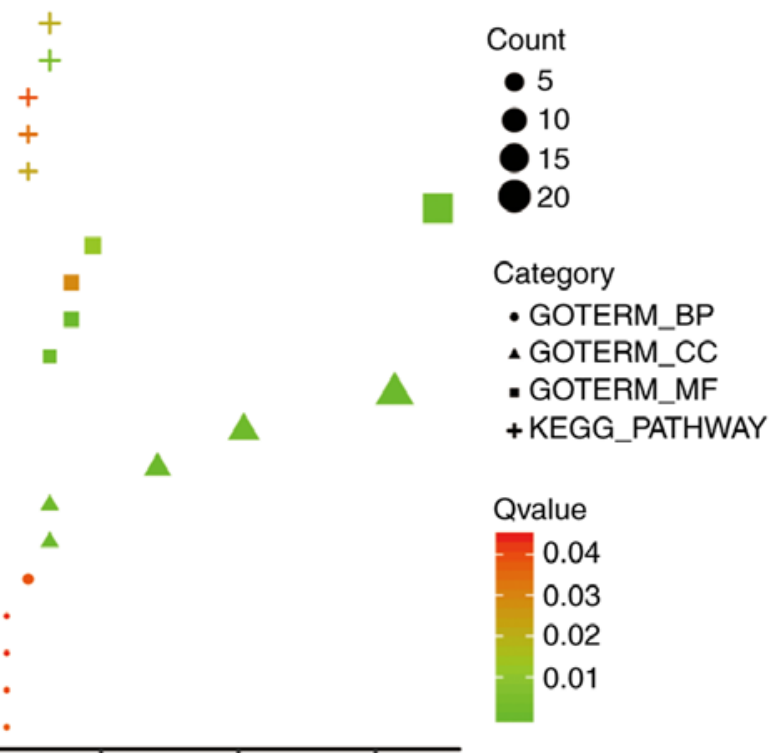

0.1

0.2

0.3

Figure 1. Bioinformatics analysis of NAFLD-related datasets. (A and B) DEGs identified from the GEO database from the datasets (A) GSE52748 and (B) GSE57425. Upregulated genes are indicated as red dots based on log fold-change $>2.0$ and $\mathrm{P}<0.05$. Downregulated genes are indicated as green dots based on $\log$ fold-change $<2.0$ and $\mathrm{P}<0.05$. Genes without significant differences are indicated as black dots. (C) Overlap analysis between gene lists and shared enriched ontologies. The Circos plot depicts the overlap between genes and functional categories based on two input gene lists derived from the DEGs. The outer circle represents the identity of the corresponding gene list, labeled GSE52748 and GSE57425, and the inner circle represents gene lists, labeled the 462 DEGs in the two data sets, where hits are arranged along the arc. Genes on the two lists are indicated in dark orange and genes unique to a list are presented as light orange. Purple curves link identical genes and blue curves link genes that belong to the same enriched ontology term. (D) Venn diagram of DEGs in the two GEO datasets. (E) GO and KEGG pathway enrichment analysis of overlapping DEGs in the two GEO datasets. GO analysis included three functional groups: Molecular functions, biological processes and cell components. DEGs, differentially expressed genes; GO, gene ontology; KEGG, Kyoto Encyclopedia of Genes and Genomes; NAFLD, non-alcoholic fatty liver disease. 

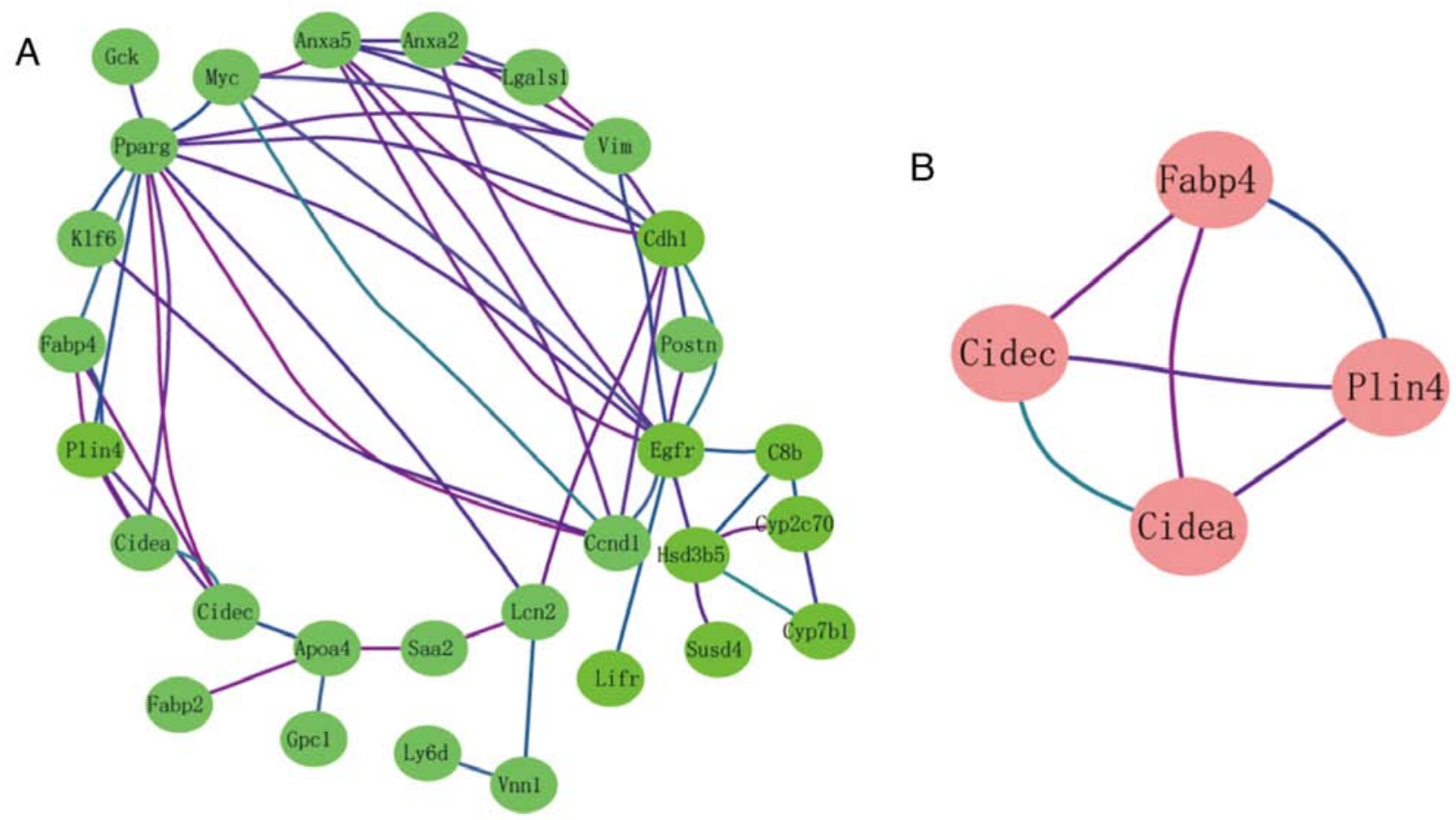

Figure 2. Module analysis of the PPI network. (A) PPI network of shared differentially expressed genes. (B) The most significant module generated from the PPI network. Cidea, cell death activator CIDE-A; Cidec, cell death activator CIDE-3; DEGs, differentially expressed gene; Fabp4, fatty acid-binding protein 4; PPI Plin4, perilipin 4, protein-protein interaction.

and washed in $0.1 \mathrm{mmol} / \mathrm{l} \mathrm{PBS}$. The sections were stained with picric acid-Sirius red $(0.1 \%$ Sirius red in saturated aqueous picric acid; Zhongshan Beijing Biotechnology Co., Ltd.) for $5 \mathrm{~min}$ at $25^{\circ} \mathrm{C}$. Slides were examined using a light microscope at magnification x 200 and $\times 400$ (Olympus Corporation). The lipid and collagen staining areas were semi-quantified using ImageJ software (version 1.8.0; National Institutes of Health).

Immunohistochemical (IHC) staining. The levels of AGE, receptor for AGE (RAGE), interleukin (IL)-1 $\beta$, IL-6 and tumor necrosis factor (TNF)- $\alpha$ in the liver tissues were measured by IHC staining in paraffin-embedded sections (thickness, $5 \mu \mathrm{m}$ ), as previously described (25). The livers were fixed in $4 \%$ paraformaldehyde solution for $24 \mathrm{~h}$ at room temperature and then dehydrated. Liver sections were deparaffinized and hydrated by sequential immersion in xylene and graded alcohol solution and heated in a microwave for $3 \mathrm{~min}$. Sections were then incubated with methanol- $3 \% \mathrm{H}_{2} \mathrm{O}_{2}$ for $10 \mathrm{~min}$ at room temperature and washed with PBS 3 times for $3 \mathrm{~min}$. Then, 20\% goat serum (cat. no. SL2-10; Beijing Solarbio Science \& Technology Co., Ltd.) was used as blocking reagent for $30 \mathrm{~min}$ at $37^{\circ} \mathrm{C}$. Sections were incubated with the following primary antibodies: Anti-AGE (1:10,000; Abcam cat. no. ab23722), anti-RAGE (1:100; Abcam, ab3611), anti-IL-1 $\beta$ (15 $\mu \mathrm{g} / \mathrm{ml}$; R\&D Systems, Inc.; cat. no. AF-401-NA), anti-IL-6 (10 $\mu \mathrm{g} / \mathrm{ml}$; R\&D Systems, Inc.; cat. no. AF-406-NA) and anti-TNF- $\alpha$ (1:300; Novus Biologicals, LLC; cat. no. NBP1-19532) overnight at $4^{\circ} \mathrm{C}$, and then incubated with the Goat Anti-Rabbit secondary antibody (1:5,000; ZB-2301; cat. no. Zhongshan Beijing Biotechnology Co., Ltd.) at $37^{\circ} \mathrm{C}$ for $30 \mathrm{~min}$. The samples were washed with PBS 3 times for $3 \mathrm{~min}$ and stained with $0.03 \%$ DAB for $5 \mathrm{~min}$ at room temperature. The slides were washed, dehydrated as above and observed using a light microscope at magnification x200 and x400 (Olympus Corporation). The positive staining area was semi-quantified using ImageJ software (version 1.8.0; National Institutes of Health) to analyze the mean optical density.

$R T-q P C R$. Total RNA was extracted from the $200 \mathrm{mg}$ liver tissues using TRNzol reagent (cat. no. DP424; Tiangen Biotech Co., Ltd.), according to the manufacturer's protocol. Total RNA was reverse transcribed into cDNA using the FastQuant RT kit (cat. no. KR106; Tiangen Biotech Co., Ltd.), according to the manufacturer's protocol. The thermocycling conditions used for qPCR were as follows: Initial denaturation at $95^{\circ} \mathrm{C}$ for $15 \mathrm{~min}$, followed by 40 cycles at $95^{\circ} \mathrm{C}$ for $10 \mathrm{sec}, 60^{\circ} \mathrm{C}$ for $20 \mathrm{sec}$ and $72^{\circ} \mathrm{C}$ for $20 \mathrm{sec}$. qPCR was performed using the SuperReal PreMix Plus kit (cat. no. FP205; Tiangen Biotech Co., Ltd.). The reactions were performed on an iCycler (Bio-Rad Laboratories, Inc.).The primer pairs used for qPCR are presented in Table SI. Relative mRNA expression was quantified using the $2^{-\Delta \Delta C q}$ method (26) and normalized to the internal reference gene Gapdh.

Statistical analysis. Statistical analysis of all results was performed using GraphPad Prism 7.0 software (GraphPad Software, Inc.). All data are presented as the mean \pm SD. Significant differences between groups were determined using an unpaired Student's t-test. $\mathrm{P}<0.05$ was considered to indicate a statistically significant difference.

\section{Results}

Identification of DEGs in NAFLD. The microarray datasets GSE52748 and GSE57425 were standardized and the results 
Table I. Functional analysis of the hub genes identified from the protein-protein interaction network.

\begin{tabular}{llrr}
\hline Term & \multicolumn{1}{c}{ Description } & Count & P-value \\
\hline GO:0005811 & Lipid particle & 3 & 0.0000000157 \\
GO:0097194 & Execution phase of apoptosis & 2 & 0.0000123000 \\
GO:0010890 & Positive regulation of sequestering of triglyceride & 1 & 0.0007238700 \\
GO:0001816 & Cytokine production & 2 & 0.0011631040 \\
GO:0010889 & Regulation of sequestering of triglyceride & 1 & 0.0012406790 \\
mmu03320 & PPAR signaling pathway & 2 & 0.0000299000
\end{tabular}

GO, Gene Ontology.

are presented in Fig. S1. A total of 106 DEGs were identified from the GSE52748 dataset, including 84 upregulated genes and 22 downregulated genes (Fig. 1A). Additionally, 390 DEGs were identified from the GSE57425 dataset, including 280 upregulated genes and 110 downregulated genes (Fig. 1B). The overlap between ontology terms associated with DEGs in GSE52748 and GSE57425 was high (Fig. 1C); thus, functional enrichment of these gene sets was analyzed together and 34 overlapping genes between the GSE52748 and GSE57425 datasets were identified (Fig. 1D).

Functional enrichment analysis of DEGs. Gene Ontology (GO) analysis identified that the DEGs were significantly enriched in cellular components, including the 'HC class II protein complex', 'extracellular exosomes', 'cell surface', 'extracellular space' and 'lipid particle' (Fig. 1E). In terms of molecular functions, DEGs were mainly enriched in 'protein phosphatase binding', 'protein binding', 'glycoprotein binding', 'protein heterodimerization activity' and 'protein kinase binding'. In addition, biological processes and Kyoto Encyclopedia of Genes and Genomes (KEGG) pathway analyses demonstrated that the DEGs were enriched in pathways involved in the 'positive regulation of glycolytic process', 'lipid transport', 'positive regulation of glycogen biosynthetic process', 'white fat cell differentiation', 'very long-chain fatty acid metabolic process', 'antigen processing and presentation', 'central carbon metabolism in cancer' and 'PPAR signaling pathway' (Fig. 1E).

Module analysis from the PPI network. The interactions of 34 DEGs were identified using the STRING online database. A PPI network was generated with Cytoscape, and the most significant modules were obtained using MCODE (Fig. 2A). Cell death-inducing DFFA-like effector a (Cidea), cell death-inducing DFFA-like effector c (Cidec), perilipin 4 (Plin4) and fatty acid-binding protein 4 (Fabp4) were identified as hub genes (Fig. 2B). These genes were closely related to the term 'regulation of sequestering triglyceride' and were enriched in the 'PPAR signaling pathway' (Table I).

Liver injury and histopathology in NASH model mice. Serum ALT and AST levels were significantly elevated in the high AGE diet group compared with the ND group (Fig. 3B). H\&E staining confirmed the presence of steatosis (Fig. 3A); the lipid content was significantly higher in the high AGE diet group compared with the ND group ( $\mathrm{P}<0.05 ;$ Fig. $3 \mathrm{C}$ and D). Collagen deposition in the liver was also elevated in the high AGE diet group compared with the ND group; however, the difference between groups was not significant $(\mathrm{P}=0.1$; Fig. $3 \mathrm{E}$ and $\mathrm{F})$. To determine whether inflammatory factors were altered in high AGE diet-induced mice, the liver concentrations of IL-1 $\beta$, IL- 6 and TNF- $\alpha$ were measured. The results revealed that the levels of these cytokines were all significantly increased in the high AGE diet group compared with the ND group (Fig. 3G-L). These results were consistent with the GSE57425 and GSE52748 datasets and suggested that mice exhibited the NASH phenotype with increased expression of inflammatory and fibrogenic factors, which is different from the NAFLD phenotype that exhibits only benign simple steatosis (27). In addition, the expression levels of AGE and RAGE in the liver were significantly elevated in the high AGE diet group compared with the ND group (Fig. 4), suggesting that the AGE/RAGE signaling pathway is involved in the pathogenesis and progression of NAFLD.

Verification of the hub genes in the NASH model mice. To validate the hub genes identified by bioinformatics analysis, RT-qPCR was performed in vivo. The expression levels of Cidea, Cidec, Fabp4 and Plin4 were significantly elevated in the liver tissues of mice fed a high AGE diet compared with those in the ND group (Fig. 5).

\section{Discussion}

The prevalence of NAFLD has increased dramatically over the last decade, surpassing alcoholic liver disease and ranking second amongst liver diseases following chronic hepatitis (28). NAFLD is closely associated with obesity and hepatic insulin resistance (29); however, the exact mechanisms of NAFLD remain largely unclear and no specific drugs for NAFLD have been approved. Thus, innovative treatment strategies are required to prevent, treat and even reverse NAFLD. Transcriptional analysis has deepened our understanding of the molecular mechanisms of human disease, which are essential to identify genetic alterations and establish potential therapeutic strategies. In the present study, two transcriptional microarray datasets, including seven NAFLD and seven normal samples were analyzed. In total, 34 overlapping DEGs were identified in the two datasets. Functional enrichment analysis revealed that the 'PPAR signaling pathway', 'central carbon metabo- 
A

HE (400x)

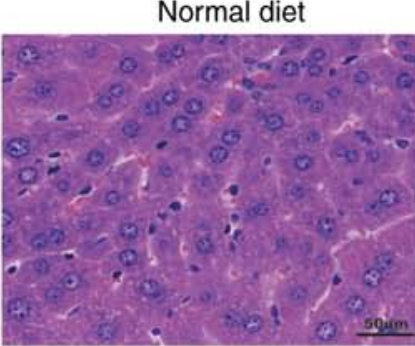

C

Oil red $\mathrm{O}$ staining

(400x)

E

Sirius red staining

(400x)

G

I

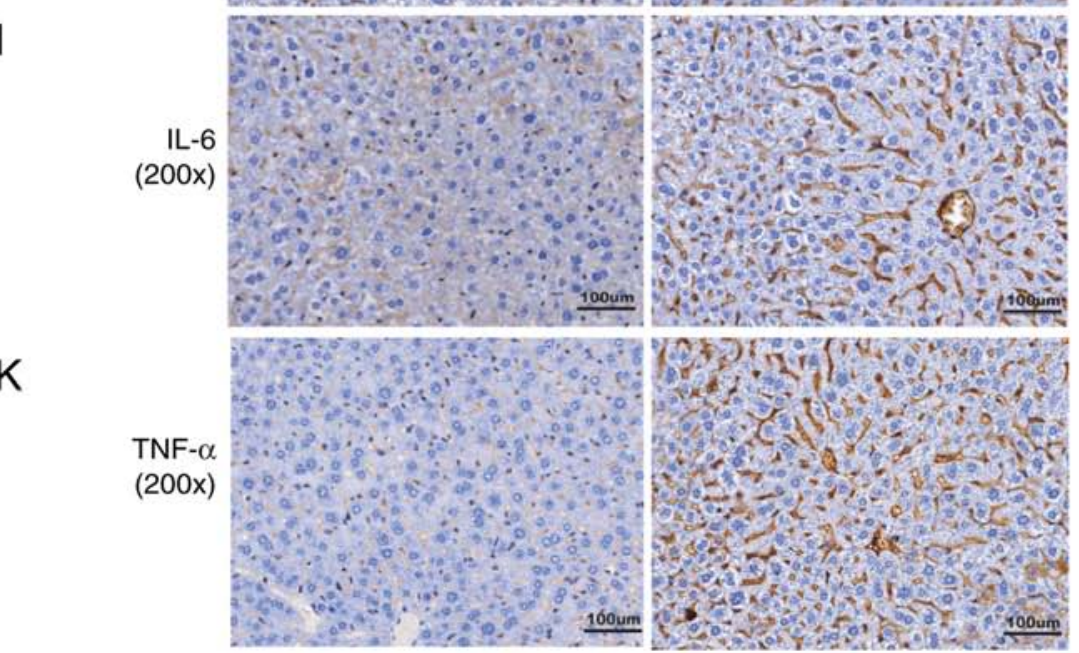

High AGEs diet
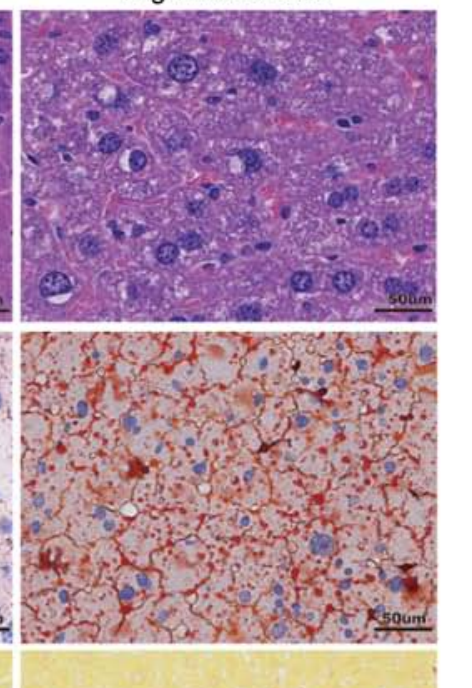

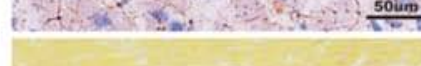

(4)
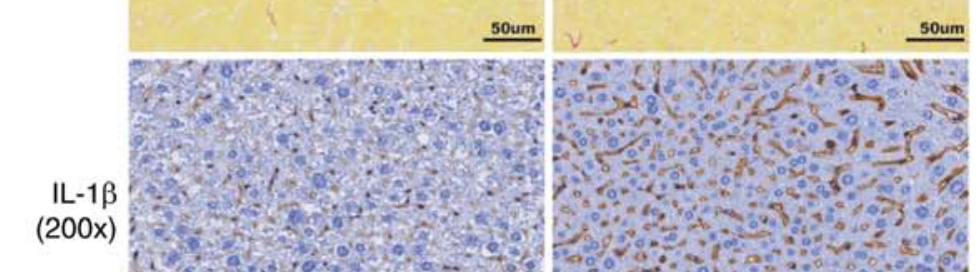

$(200 x)$
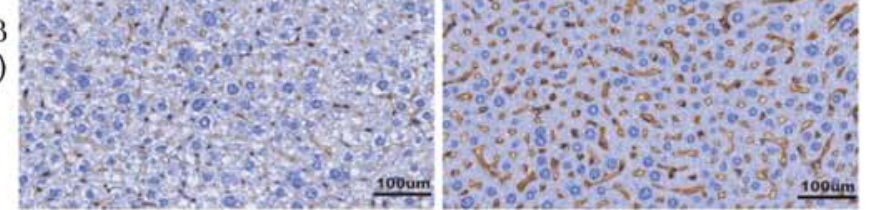

$160 u m$
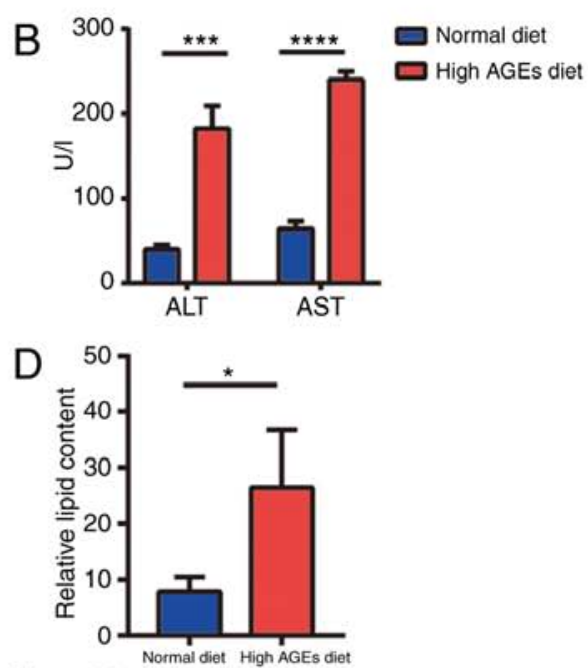

$\mathrm{F}$

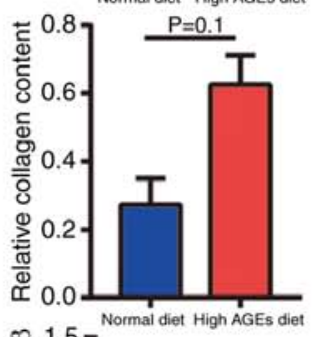

$\mathrm{H}$

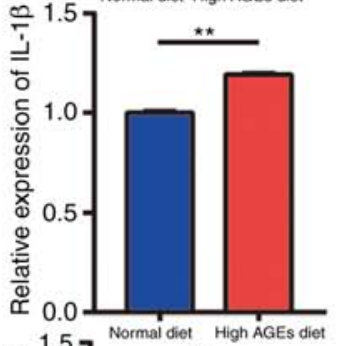

$J$

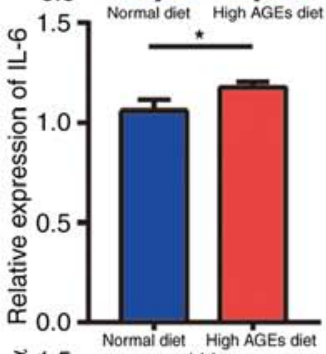

$\mathrm{L}$

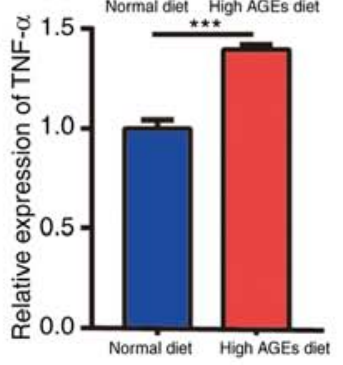

Figure 3. Representative micrographs of liver injury and histopathology in NASH model mice. (A) Hematoxylin \& eosin staining of mice livers (magnification, x400). (B) Serum levels of ALT and AST. (C) Oil red O staining of mice livers and (D) semi-quantification of the lipid content. (E) Sirius red staining of mice livers (magnification, $\mathrm{x} 400$ ) and (F) semi-quantification of the collagen deposition. (G-K) Representative micrographs (magnification, x200) and semi-quantification of protein expression levels of (G and H) IL-1 $\beta$, (I and J) IL-6 and (K and L) TNF- $\alpha$ in liver tissues. ${ }^{*} \mathrm{P}<0.05,{ }^{* *} \mathrm{P}<0.01,{ }^{* * * *} \mathrm{P}<0.001$ and ${ }^{* * * * *} \mathrm{P}<0.0001$. AGE, advanced glycation end-product; ALT, alanine aminotransferase; AST, aspartate transaminase; HE, hematoxylin \& eosin; IL, interleukin; $\mathrm{ND}$, normal diet; TNF, tumor necrosis factor.

lism in cancer' and 'cell adhesion molecules (CAMs)' are involved in NAFLD. Moreover, four hub genes (Cidea, Cidec, Fabp4 and Plin4) were identified from the PPI network. Upon experimentally verifying the four hub genes by RT-qPCR, the expression levels of these genes in the NASH model mice were consistent with the results from the bioinformatics analysis. 
A

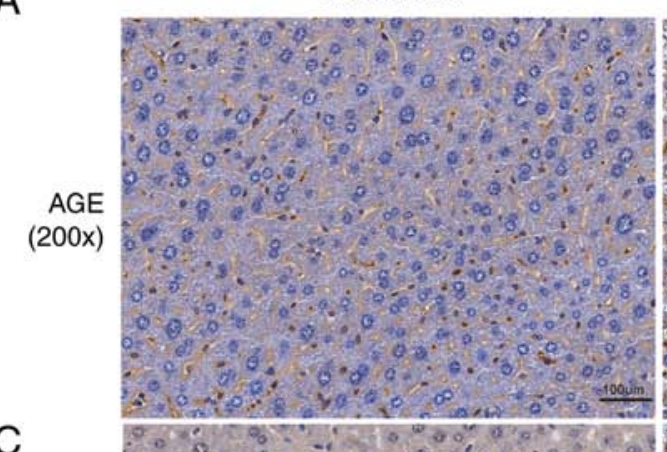

C

RAGE $(200 x)$

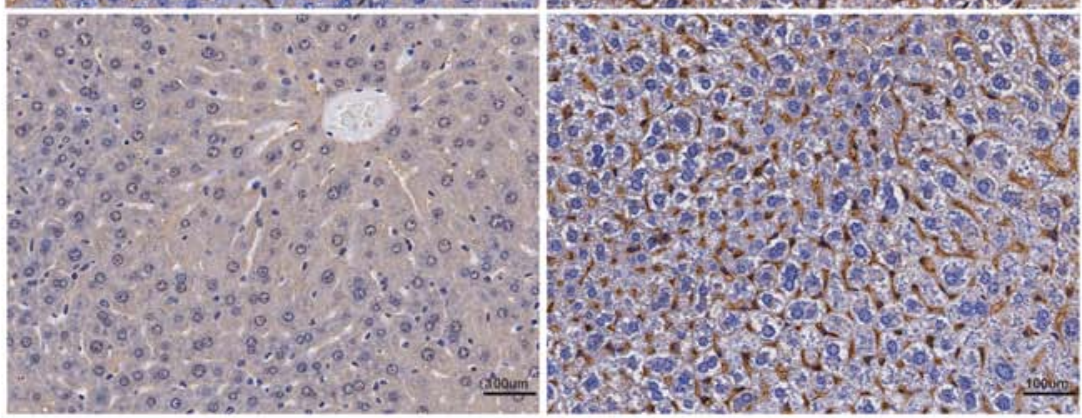

High AGEs diet

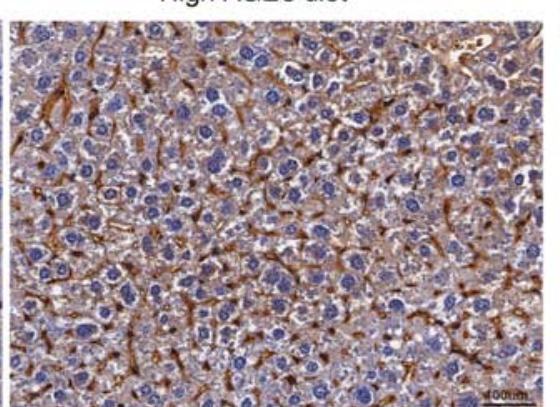

D
B
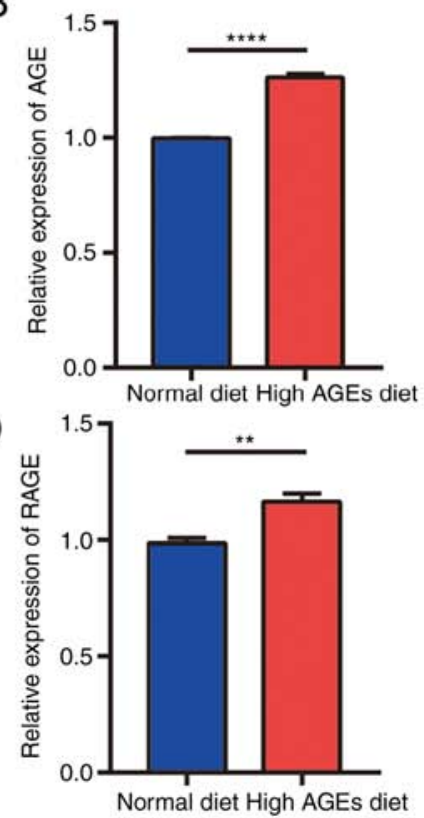

Figure 4. Determination of the expression levels of AGE and RAGE in NASH model mice using immunohistochemical staining. (A) Representative micrographs (magnification, x200) and (B) semi-quantification of the protein expression levels of AGE in liver. (C) Representative micrographs (magnification, $\mathrm{x} 200$ ) and (D) semi-quantification of the protein expression levels of RAGE in liver tissues. Scale bars, $100 \mu \mathrm{m} .{ }^{* * *} \mathrm{P}<0.01$ and ${ }^{* * * * *} \mathrm{P}<0.0001$. AGE, advanced glycation end-product; ND, normal diet; RAGE, receptor of advanced glycation end-product.
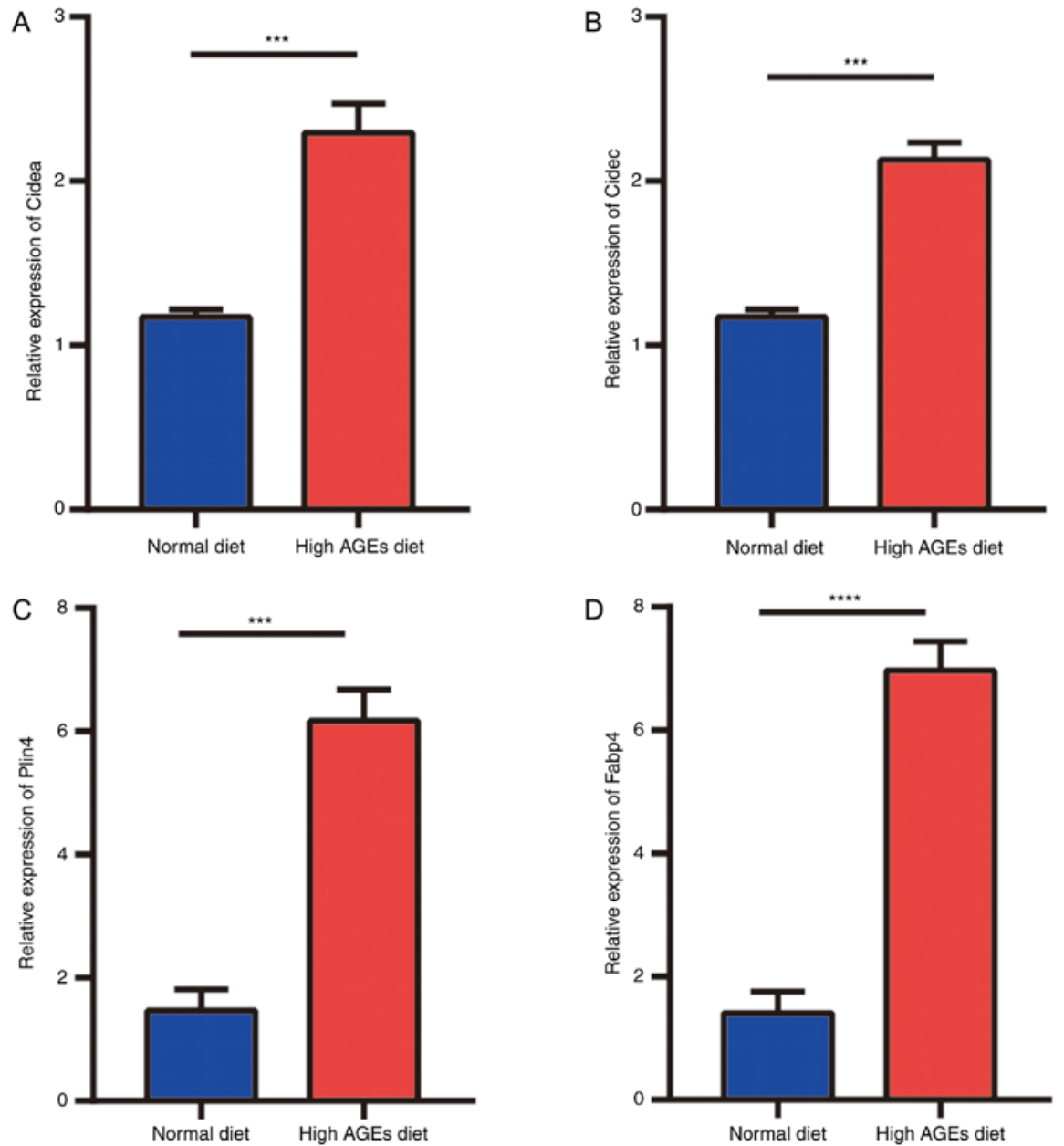

Figure 5. Expression levels of hub genes in NASH model mice, assessed by reverse transcription-quantitative PCR. Expression levels of (A) Cidea, (B) Cidec, (C) Plin4 and (D) Fabp4 in liver tissues. ${ }^{* * *} \mathrm{P}<0.001$ and ${ }^{* * * * *} \mathrm{P}<0.0001$. AGE, advanced glycation end-product; Cidea, cell death-inducing DFFA-like effector a; Cidec, cell death-inducing DFFA-like effector c; Fabp4, fatty acid-binding protein 4; Plin4, perilipin 4. 
NASH is the second leading etiological factor of liver disease among adults waiting for liver transplantations and it is mostly commonly managed through lifestyle interventions combined with pharmacological interventions (28). Thus, the NASH model is widely used to explore pharmacological strategies for liver disease (28).

Previous studies have demonstrated that PPARs serve an essential role in metabolic signaling networks and inflammation, and are important regulators of the pathogenesis of NAFLD (30-32). PPARs exist as three isotypes (PPAR $\alpha$, PPAR $\beta / \delta$ and PPAR $\gamma$ ), which have various tissue expression patterns and specificities. PPAR $\alpha$, which is highly expressed in the liver, serves hepatoprotective roles through mediating mitochondrial functions, and exhibiting anti-inflammatory and antifibrotic effects $(33,34)$. PPAR $\beta / \delta$, mainly expressed in the gastrointestinal tract, heart and kidney, can improve hepatic steatosis through activating fatty acid $\beta$-oxidation and reducing lipogenesis $(35,36)$. PPAR $\gamma$, which is highly expressed in adipose tissues, has an important role in transcription and glucose metabolism (37). Thus, PPAR modulators, including dual or pan-PPAR agonists represent potential as therapeutic targets in NAFLD.

Four hub genes (Cidea, Cidec, Fabp 4 and Plin4) were identified as having the highest scores in the PPI network. Cidea and Cidec, which belong to a family of cell death-inducing DNA fragmentation factor-a-like effector proteins, serve important roles in hepatic lipid metabolism (38). Additionally, numerous studies have revealed that Cidea and Cidec protein expression levels were highly elevated in the liver of mice fed HFD $(39,40)$. FABP4, an intracellular lipid transporter, has a prominent role in lipid-mediated biological processes and systemic metabolic homeostasis (41). PLIN4 is a known PPAR $\gamma$ target, which is involved in the pathophysiology of NAFLD (42). Carino et al (43) reported that the expression levels of Cidec, Cidea and Plin4 were increased in NASH model mice, according to transcriptome analysis. In accordance with previous studies, the results of the present study revealed that the expression levels of Cidea, Cidec, Fabp 4 and Plin4 were significantly higher in the livers of NASH model mice fed a high AGE diet compared with those in the livers of mice in the ND group. CIDEA, CIDEC and PLIN4 localize to lipid droplets to promote lipid droplet fusion and hepatic lipid storage under high caloric intake, thus promoting liver steatosis $(42,44)$. FABP4 is highly upregulated by fatty acids and inflammatory activation, and further promotes lipid infusion and inflammation in hepatocytes (45). Thus, these results suggested that the identified hub genes may be used as therapeutic targets of NAFLD.

In addition, the present study demonstrated that the NASH model mice were in a state of hepatic steatosis, inflammation and fibrosis following the administration of a high AGE diet. Collagen deposits were not significantly increased compared with the ND group, which may be due to the inadequate AGE content in the various food sources and preparation methods carried out in the present study. Notably, RAGE expression levels were significantly increased in high AGE diet mice, suggesting a role for the AGE/RAGE axis in NAFLD. It is well known that AGEs promote liver injury, inflammation and fibrosis through their interaction with RAGE, which in turn activates oxidative and inflammatory events, creating a positive feedback loop (46). Thus, these results suggested that targeting the AGE/RAGE pathway may be an effective therapeutic strategy for treating NASH. In addition, previous studies have highlighted the effects of dietary AGEs on the gut microbiota and their ability to cause metabolic diseases, including NAFLD $(47,48)$. Owing to the increasing prevalence of western diets, alterations in the microbiome by dietary AGEs are of particular interest (49); thus, this may represent a promising therapeutic target.

In conclusion, the findings of the present study revealed that high dietary AGEs can induce liver injury, inflammation and even hepatic fibrosis. Additionally, four hub genes involved in NAFLD progression were identified. These results suggested that the restriction of dietary AGEs or pharmacological strategies targeting the four hub genes may represent novel approaches for treating and preventing NAFLD.

\section{Acknowledgements}

Not applicable.

\section{Funding}

The present study was supported by grants from The National Natural Science Funds of China (grant nos. 81760168 and 81560154) and The Innovation Fund Project in Jiangxi Province (grant no. YC2016-S100).

\section{Availability of data and materials}

The datasets used and/or analyzed during the present study are available from the corresponding author on reasonable request.

\section{Authors' contributions}

JW performed the animal study and wrote the manuscript. HHL performed the histological examination of the liver. GJX performed the data analysis. WC performed the RT-qPCR analysis. JXX conceived and designed the study. All authors read and approved the final manuscript.

\section{Ethics approval and consent to participate}

The experimental protocol of this study was approved by the Research Committee of the First Affiliated Hospital of Nanchang University (Nanchang, China).

\section{Patient consent for publication}

Not applicable.

\section{Competing interests}

The authors declare that they have no competing interests.

\section{References}

1. Estes C, Razavi H, Loomba R, Younossi Z and Sanyal AJ: Modeling the epidemic of nonalcoholic fatty liver disease demonstrates an exponential increase in burden of disease. Hepatology 67: 123-133, 2018. 
2. Yki-Järvinen H: Non-alcoholic fatty liver disease as a cause and a consequence of metabolic syndrome. Lancet Diabetes Endocrinol 2: 901-910, 2014.

3. Gluchowski NL, Becuwe M, Walther TC and Farese RV Jr: Lipid droplets and liver disease: From basic biology to clinical implications. Nat Rev Gastroenterol Hepatol 14: 343-355, 2017.

4. Trovato FM, Castrogiovanni P, Malatino L and Musumeci G: Nonalcoholic fatty liver disease (NAFLD) prevention: Role of Mediterranean diet and physical activity. Hepatobiliary Surg Nutr 8: 167-169, 2019.

5. Trovato FM, Martines GF, Brischetto D, Catalano D, Musumeci G and Trovato GM: Fatty liver disease and lifestyle in youngsters: Diet, food intake frequency, exercise, sleep shortage and fashion. Liver Int 36: 427-433, 2016.

6. Trovato FM, Catalano D, Musumeci G and Trovato GM: 4Ps medicine of the fatty liver: The research model of predictive, preventive, personalized and participatory medicine-recommendations for facing obesity, fatty liver and fibrosis epidemics. EPMA J 5: 21, 2014

7. Singh R, Barden A, Mori T and Beilin L: Advanced glycation end-products: A review. Diabetologia 44: 129-146, 2001.

8. Uribarri J, Woodruff S, Goodman S, Cai W, Chen X, Pyzik R, Yong A, Striker GE and Vlassara H: Advanced glycation end products in foods and a practical guide to their reduction in the diet. J Am Diet Assoc 110: 911-916.e12, 2010.

9. Leung C,HerathCB,JiaZ, Goodwin M,Mak KY, Watt MJ,Forbes JM and Angus PW: Dietary glycotoxins exacerbate progression of experimental fatty liver disease. J Hepatol 60: 832-838, 2014.

10. Ravichandran G, Lakshmanan DK, Raju K, Elangovan A Nambirajan G, Devanesan AA and Thilagar S: Food advanced glycation end products as potential endocrine disruptors: An emerging threat to contemporary and future generation. Environ Int 123: 486-500, 2019.

11. Chaudhuri J, Bains Y, Guha S, Kahn A, Hall D, Bose N, Gugliucci A and Kapahi P: The role of advanced glycation end products in aging and metabolic diseases: Bridging association and causality. Cell Metab 28: 337-352, 2018.

12. Zhang X, Wang Y and Liu P: Omic studies reveal the pathogenic lipid droplet proteins in non-alcoholic fatty liver disease. Protein Cell 8: 4-13, 2017

13. Kahle M, Horsch M, Fridrich B, Seelig A, Schultheiß J, Leonhardt J, Irmler M, Beckers J, Rathkolb B, Wolf E, et al: Phenotypic comparison of common mouse strains developing high-fat diet-induced hepatosteatosis. Mol Metab 2: 435-446, 2013.

14. Zhou D, Hlady RA, Schafer MJ, White TA, Liu C, Choi JH, Miller JD, Roberts LR, LeBrasseur NK and Robertson KD: High fat diet and exercise lead to a disrupted and pathogenic DNA methylome in mouse liver. Epigenetics 12: 55-69, 2017.

15. Pruis MG, Lendvai A, Bloks VW, Zwier MV, Baller JF, de Bruin A, Groen AK and Plösch T: Maternal western diet primes non-alcoholic fatty liver disease in adult mouse offspring. Acta Physiol (Oxf) 210: 215-227, 2014.

16. Dorn C, Engelmann JC, Saugspier M, Koch A, Hartmann A Müller M, Spang R, Bosserhoff A and Hellerbrand C: Increased expression of c-Jun in nonalcoholic fatty liver disease. Lab Invest 94: 394-408, 2014.

17. Lu Y, Liu X, Jiao Y, Xiong X, Wang E, Wang X, Zhang Z, Zhang H, Pan L, Guan Y, et al: Periostin promotes liver steatosis and hypertriglyceridemia through downregulation of PPAR $\alpha$. J Clin Invest 124: 3501-3513, 2014.

18. Davis S and Meltzer PS: GEOquery: A bridge between the gene expression omnibus (GEO) and BioConductor. Bioinformatics 23 : $1846-1847,2007$

19. Yu Y, Ouyang Y and Yao W: shinyCircos: An R/Shiny application for interactive creation of Circos plot. Bioinformatics 34 1229-1231, 2018

20. Smoot ME, Ono K, Ruscheinski J, Wang PL and Ideker T: Cytoscape 2.8: New features for data integration and network visualization. Bioinformatics 27: 431-432, 2011.

21. Sourris KC, Harcourt BE, Penfold SA, Yap FY, Morley AL, Morgan PE, Davies MJ, Baker ST, Jerums G and Forbes JM Modulation of the cellular expression of circulating advanced glycation end-product receptors in type 2 diabetic nephropathy. Exp Diabetes Res 2010: 974681, 2010

22. Kleiner DE, Brunt EM, Van Natta M, Behling C, Contos MJ, Cummings OW, Ferrell LD, Liu YC, Torbenson MS Unalp-Arida A, et al: Design and validation of a histological scoring system for nonalcoholic fatty liver disease. Hepatology 41 : $1313-1321,2005$
23. Mehlem A, Hagberg CE, Muhl L, Eriksson U and Falkevall A Imaging of neutral lipids by oil red $\mathrm{O}$ for analyzing the metabolic status in health and disease. Nat Protoc 8: 1149-1154, 2013.

24. Goodwin M, Herath C, Jia Z, Leung C, Coughlan MT, Forbes J and Angus P: Advanced glycation end products augment experimental hepatic fibrosis. J Gastroenterol Hepatol 28: 369-376, 2013.

25. Trovato FM, Castrogiovanni P, Szychlinska MA, Purrello F and Musumeci G: Early effects of high-fat diet, extra-virgin olive oil and vitamin $\mathrm{D}$ in a sedentary rat model of non-alcoholic fatty liver disease. Histol Histopathol 33: 1201-1213, 2018.

26. Livak KJ and Schmittgen TD: Analysis of relative gene expression data using real-time quantitative PCR and the 2(-Delta Delta C(T)) method. Methods 25: 402-408, 2001.

27. Farrell GC and Larter CZ: Nonalcoholic fatty liver disease: From steatosis to cirrhosis. Hepatology 43 (2 Suppl 1): S99-S112, 2006

28. Wong RJ, Aguilar M, Cheung R, Perumpail RB, Harrison SA, Younossi ZM and Ahmed A: Nonalcoholic steatohepatitis is the second leading etiology of liver disease among adults awaiting liver transplantation in the United States. Gastroenterology 148: 547-555, 2015.

29. Samuel VT and Shulman GI: Nonalcoholic fatty liver disease as a nexus of metabolic and hepatic diseases. Cell Metab 27: 22-41, 2018.

30. Pawlak M, Lefebvre P and Staels B: Molecular mechanism of PPAR $\alpha$ action and its impact on lipid metabolism, inflammation and fibrosis in non-alcoholic fatty liver disease. J Hepatol 62: 720-733, 2015.

31. Gross B, Pawlak M, Lefebvre P and Staels B: PPARs in obesity-induced T2DM, dyslipidaemia and NAFLD. Nat Rev Endocrinol 13: 36-49, 2017.

32. Liss KH and Finck BN: PPARs and nonalcoholic fatty liver disease. Biochimie 136: 65-74, 2017.

33. Pawlak M, Baugé E, Bourguet W, De Bosscher K, Lalloyer F, Tailleux A, Lebherz C, Lefebvre P and Staels B: The transrepressive activity of peroxisome proliferator-activated receptor alpha is necessary and sufficient to prevent liver fibrosis in mice. Hepatology 60: 1593-1606, 2014.

34. Koliaki C, Szendroedi J, Kaul K, Jelenik T, Nowotny P, Jankowiak F, Herder C, Carstensen M, Krausch M, Knoefel WT, et al: Adaptation of hepatic mitochondrial function in humans with non-alcoholic fatty liver is lost in steatohepatitis. Cell Metab 21: 739-746, 2015.

35. Wu HT, Chen CT, Cheng KC, Li YX, Yeh CH and Cheng JT: Pharmacological activation of peroxisome proliferator-activated receptor $\delta$ improves insulin resistance and hepatic steatosis in high fat diet-induced diabetic mice. Horm Metab Res 43: 631-635, 2011.

36. Bojic LA, Telford DE, Fullerton MD, Ford RJ, Sutherland BG, Edwards JY, Sawyez CG, Gros R, Kemp BE, Steinberg GR and Huff MW: PPAR $\delta$ activation attenuates hepatic steatosis in Ldlr-/- mice by enhanced fat oxidation, reduced lipogenesis, and improved insulin sensitivity. J Lipid Res 55: 1254-1266, 2014.

37. Bensinger SJ and Tontonoz P: Integration of metabolism and inflammation by lipid-activated nuclear receptors. Nature 454 : 470-477, 2008

38. Xu L, Zhou L and Li P: CIDE proteins and lipid metabolism. Arterioscler Thromb Vasc Biol 32: 1094-1098, 2012

39. Zhou L, Xu L, Ye J, Li D, Wang W, Li X, Wu L, Wang H, Guan F and Li P: Cidea promotes hepatic steatosis by sensing dietary fatty acids. Hepatology 56: 95-107, 2012.

40. Reynolds TH IV, Banerjee S, Sharma VM, Donohue J, Couldwell S, Sosinsky A, Frulla A, Robinson A and Puri V: Effects of a high fat diet and voluntary wheel running exercise on cidea and cidec expression in liver and adipose tissue of mice. PLoS One 10: e0130259, 2015.

41. Hotamisligil GS and Bernlohr DA: Metabolic functions of FABPs-mechanisms and therapeutic implications. Nat Rev Endocrinol 11: 592-605, 2015.

42. Carr RM and Ahima RS: Pathophysiology of lipid droplet proteins in liver diseases. Exp Cell Res 340: 187-192, 2016.

43. Carino A, Marchianò S, Biagioli M, Fiorucci C, Zampella A Monti MC, Morretta E, Bordoni M, Di Giorgio C, Roselli R, et al: Transcriptome analysis of Dual FXR and GPBAR1 agonism in rodent model of NASH reveals modulation of lipid droplets formation. Nutrients 11: pii: E1132, 2019.

44. Slayton M, Gupta A, Balakrishnan B and Puri V: CIDE proteins in human health and disease. Cells 8: pii: E238, 2019.

45. Thompson KJ, Austin RG, Nazari SS, Gersin KS, Iannitti DA and McKillop IH: Altered fatty acid-binding protein 4 (FABP4) expression and function in human and animal models of hepatocellular carcinoma. Liver Int 38: 1074-1083, 2018. 
46. Leung C, Herath CB, Jia Z, Andrikopoulos S, Brown BE, Davies MJ, Rivera LR, Furness JB, Forbes JM and Angus PW: Dietary advanced glycation end-products aggravate non-alcoholic fatty liver disease. World J Gastroenterol 22: 8026-8040, 2016.

47. Delgado-Andrade C, Pastoriza de la Cueva S, Peinado MJ, Rufián-Henares JÁ, Navarro MP and Rubio LA: Modifications in bacterial groups and short chain fatty acid production in the gut of healthy adult rats after long-term consumption of dietary Maillard reaction products. Food Res Int 100: 134-142, 2017.

48. Qu W, Yuan X, Zhao J, Zhang Y, Hu J, Wang J and Li J: Dietary advanced glycation end products modify gut microbial composition and partially increase colon permeability in rats. Mol Nutr Food Res 61, 2017
49. Snelson M and Coughlan MT: Dietary advanced glycation end products: Digestion, metabolism and modulation of gut microbial ecology. Nutrients 11: pii: E215, 2019.

(i) (3) This work is licensed under a Creative Commons

EY NC NO Attribution-NonCommercial-NoDerivatives 4.0 International (CC BY-NC-ND 4.0) License. 